VOL. $72(2005)$ [225-250]

\title{
ABSTRACT THEORY OF SEMIORDERINGS
}

\author{
Thomas C. Craven and Tara L. Smith
}

\begin{abstract}
Marshall's abstract theory of spaces of orderings is a powerful tool in the algebraic theory of quadratic forms. We develop an abstract theory for semiorderings, developing a notion of a space of semiorderings which is a prespace of orderings. It is shown how to construct all finitely generated spaces of semiorderings. The morphisms between such spaces are studied, generalising the extension of valuations for fields into this context. An important invariant for studying Witt rings is the covering number of a preordering. Covering numbers are defined for abstract preorderings and related to other invariants of the Witt ring.
\end{abstract}

\section{INTRODUCTION}

Some of the earliest work with formally real fields in quadratic form theory, following Pfister's revitalisation of this area of research, involved Witt rings of equivalence classes of nondegenerate quadratic forms and the spaces of orderings which are closely associated with the prime ideals of the Witt rings. A ring theoretic approach was pioneered by Knebusch, Rosenberg and Ware in [20]. The spaces of orderings were studied in [6] and all Boolean spaces were shown to occur. A closer tie to the theory of quadratic forms was achieved by $M$. Marshall who developed an abstract theory of spaces of orderings to help in studying the reduced Witt rings of formally real fields (see [22]). Marshall's theory was more closely related to what actually happens with Witt rings of fields than the work in [20] (and this was later put into a ring-theoretic context by Rosenberg and Kleinstein [18]). Since that time, Marshall's work has been used by numerous authors; the abstract approach has been shown to provide a common way of proving theorems for not just fields, but also semilocal rings [17] and *-fields [11]. In spite of all the work in this area, there are still major open questions, most notably those of realisability of abstract spaces and realisability of images of Witt rings. The theory of abstract spaces of orderings has also been generalised in many ways, in particular to (nonreduced) Witt rings of a field (see [22]) and to real algebraic geometry $[23,1]$.

In this paper, the authors move the generalisation in another direction. They create an abstract theory for semiorderings of a field. It is also known to apply to *-semiorderings on a *-field [10]. The motivation for this is two-fold. First of all, semiorderings are

Received 31st March, 2005

Copyright Clearance Centre, Inc. Serial-fee code: 0004-9727/05 \$A2.00+0.00. 
important in their own right for fields, where they were studied in the early 1970s by Prestel (see [25]) with regard to axiom systems in geometry and quadratic form questions. The initial work characterised the situation in which every semiordering was actually an ordering. Those results were extended to semilocal rings $[17,19]$. More recently, Prestel and others $[16,26]$ have made major use of the concept in the rather concrete study of positive polynomials in polynomial rings. Other uses in field theory can be found in $[4,3,14,21]$; in particular, Bröcker [4] develops the field version of Theorem 1.6 and relates it to the computation of the stability index of a field. A totally different occurrence of semiorderings begins with the work of $R$. Baer [2]. Baer orderings of a *-field give an example of a noncommutative generalisation of semiordering for which very little is known concerning the Witt groups that arise (see [11] for a discussion of various generalisations to *-fields). This more general context will be taken up briefly in Section 2. For the more restrictive concepts of *-ordering and *-semiordering, the work of [10] shows that the hermitian form structure as determined by Witt rings has exactly the same properties as when $*$ is the identity. One purpose of the present paper is to unify the semiordering work of these various contexts to a single more abstract setting. The authors hope to use the present work to further both the commutative and noncommutative directions in their future work.

The remainder of this section provides the abstract definition and some reasons for believing that it is the correct one. In addition to a special setting for generalising Baer orderings, Section 2 introduces three operations for building new spaces of semiorderings from old ones. Section 3 generalises the lifting theorems of Prestel from the context of valuations on fields to our abstract setting. We also look at morphisms on spaces of semiorderings, again motivated by valuation theoretic results for fields. Section 4 carries this abstract theory to preorderings and covering numbers. This was the original motivation for this paper, stemming from work in [14]. Theorems 4.4 and 4.8 are the main ones of the section and are aimed at elucidating the connection between semiorderings and preorderings under group extensions (corresponding to lifting results from the residue field in the case of valuations). Section 5 and the Appendix take a brief look at certain invariants based on semiorderings when applied to finite spaces of orderings.

DEFINITION 1.1: ([23, p. 23]) A space of orderings is a pair $(X, G)$ satisfying axioms $\mathrm{AX} 1, \mathrm{AX} 2, \mathrm{AX} 3$ below.

AX1. $X$ is a nonempty set, $G$ is a subgroup of $\{-1,1\}^{X}$ (the set of all functions $X \rightarrow\{ \pm 1\}$ with its natural group structure), $G$ contains the constant function -1 and $G$ separates points in $X$.

Note that we can identify $X$ with a subset of the character group $\chi(G) \subseteq\{-1,1\}^{c}$, consisting of all homomorphisms $\sigma: G \rightarrow\{ \pm 1\}$, via $x(g)=g(x)$ since $G$ separates points of $X$. For $a, b \in G$, we define the value set $D\langle a, b\rangle$ to be the set of all $c \in G$ such that for each $x \in X$, either $c(x)=a(x)$ or $c(x)=b(x)$. 
AX2. If $x \in \chi(G)$ satisfies $x(-1)=-1$ and

$$
a, b \in \operatorname{ker} x \Longrightarrow D\langle a, b\rangle \subseteq \operatorname{ker} x,
$$

then $x$ is in the image of the natural embedding of $X$ into $\chi(G)$.

AX3. For all $g_{1}, g_{2}, g_{3} \in G$, if $h \in D\left\langle g_{1}, c\right\rangle$ for some $c \in D\left\langle g_{2}, g_{3}\right\rangle$, then $h \in D\left\langle d, g_{3}\right\rangle$ for some $d \in D\left\langle g_{1}, g_{2}\right\rangle$.

The motivation for this definition is the example of a formally real field $F$ with $G$ equal to $\dot{F} / \Sigma \dot{F}^{2}$, where $\Sigma \dot{F}^{2}$ denotes the group of sums of nonzero squares in the field $F$, and $X$ equal to the space of orderings of $F$. For $a, b \in \dot{F}$, the value set is

$$
D\langle a, b\rangle=\left\{a x^{2}+b y^{2} \in \dot{F} \mid x, y \in F\right\} .
$$

Now AX2 essentially gives the definition of the positive cone of an ordering and the hypothesis of AX3 says that $h$ has the form $h=a_{1} g_{1}^{2}+a_{2} g_{2}^{2}+a_{3} g_{3}^{2}$, from which we see that $d=a_{1} g_{1}^{2}+a_{2} g_{2}^{2}$ works.

Definition 1.2: Let $(X, G)$ be a space of orderings. We define a space of semiorderings for $(X, G)$ to be the pair $(Y, G)$ where $Y$ is given by

$$
\begin{aligned}
Y=\left\{y \in\{-1,1\}^{G} \mid y(1)=1, \quad y(-g)=\right. & -y(g) \forall g \in G, \\
& \text { and } a, b \in \operatorname{ker} y \Longrightarrow D\langle a, b\rangle \subseteq \operatorname{ker} y\} .
\end{aligned}
$$

Note that $X \subseteq Y$ since the elements of $X$ are required to satisfy condition (1.1). The topology on $Y$ is that induced by the product topology on $\{-1,1\}^{G}$. This is easily seen to be the same as the topology with subbasis consisting of sets of the form

$$
H(g)=\{y \in Y \mid g(y)=1\}
$$

for any $g \in G$. We shall refer to this subbasis as the Harrison subbasis as is usually done when $Y$ is replaced by $X$. Notice that the Harrison subbasis can be viewed as a group isomorphic to $\mathrm{G}$; indeed, when the sets are restricted to $X$, we have the symmetric difference $H(-a)+H(-b)=H(-a b)$. (However, see the example following Definition 2.1 for the situation with Baer orderings of *-fields.) The basis for the topology consists of finite intersections of the form

$$
H\left(a_{1}, \ldots, a_{n}\right)=\bigcap_{i=1}^{n} H\left(a_{i}\right)
$$

In the case of $(X, G)$, there is a third way of viewing the topology, namely as the Zariski topology on the space of minimal prime ideals of the Witt ring, defined below. We shall see in the next section that this third method can also be used for $Y$; in particular, $Y$, like $X$, is a Boolean space (compact, Hausdorff and totally disconnected). 
Proposition 1.3. Given a space of semiorderings $(Y, G)$ for the space of orderings $(X, G)$, the value sets $D\langle a, b\rangle$ satisfy $c \in D\langle a, b\rangle$ if and only if

$$
c(y) \in\{a(y), b(y)\} \forall y \in Y
$$

PROOF: One direction follows immediately from the fact that $Y \supseteq X$. The reverse direction follows from the definition if $a(y)=b(y)=1$ and is trivial if they have opposite signs. If $a(y)=b(y)=-1$, then $y(-a)=y(-b)=1$ and $-c \in D\langle-a,-b\rangle$, so $y(-c)=1$, giving $y(c)=-1$.

In the case of fields, the space of orderings and space of semiorderings uniquely determine one another. This is also easily seen in the abstract setting, as the next proposition shows.

Proposition 1.4. A space of semiorderings $(Y, G)$ uniquely determines the space of orderings $X \subseteq Y$.

Proof: Assume a fixed $(Y, G)$. We claim that the space $X$ of the definition is actually the unique maximal subset of $Y$ such that $\left.G\right|_{X}=\left\{\left.g\right|_{X} \mid g \in G\right\}$ is a subgroup of $\{ \pm 1\}^{X}$. The uniqueness of the maximal subset follows from the fact that if $\left.G\right|_{X_{1}}$ and $\left.G\right|_{X_{2}}$ are groups, so is $\left.G\right|_{X_{1} \cup X_{2}}$. The fact that $X$ must be maximal follows from axiom AX2 which forces elements into $X$ : Since every element of $X$ must satisfy

$$
a, b \in \operatorname{ker} x \Longrightarrow D\langle a, b\rangle \subseteq \operatorname{ker} x
$$

the set $X$ is uniquely determined as a subset of $\chi(G)$.

We provide a brief description of the Witt ring $W(X, G)$ associated with a space of orderings. For more details, see [22] [23, Section 2.2]. The ring $W(X, G)$ consists of equivalence classes of formally defined forms $\varphi=\left\langle a_{1}, a_{2}, \ldots, a_{m}\right\rangle$, where $a_{i} \in G$. The number $m$ is called the dimension of $\varphi$, written $m=\operatorname{dim} \varphi$. If $\varphi=\left\langle a_{1}, a_{2}, \ldots, a_{m}\right\rangle$ and $\psi=\left\langle b_{1}, b_{2}, \ldots, b_{n}\right\rangle$, addition is defined by

$$
\varphi \oplus \psi=\left\langle a_{1}, a_{2}, \ldots, a_{m}, b_{1}, b_{2}, \ldots, b_{n}\right\rangle
$$

Multiplication is extended via the distributive law from $\langle a\rangle \otimes\langle b\rangle=\langle a b\rangle$ for $a, b \in G$. We can view the form $\varphi$ as a function from $X$ to $\mathbb{Z}$ via $\varphi(x)=\sum_{i=1}^{m} a_{i}(x)$. We shall call the image in $\mathbb{Z}$ the signature of $\varphi$. Using this, we have $\varphi$ and $\psi$ are equal as elements of $W(X, G)$ (written $\varphi \sim \psi$ ) if and only if $\varphi(x)=\psi(x)$ for all $x \in X$ [23, Theorem 2.3.1]. We also use the concept of isometry, writing $\varphi \cong \psi$ if $\varphi$ and $\psi$ have the same dimension and signature [23, Theorem 2.2.3]. For the purpose of ease in writing proofs, it is more useful to have the following definition of isometry: for 1-dimensional forms $\langle a\rangle \cong\langle b\rangle$ means $a=b$. For 2-dimensional forms, $\left\langle a_{1}, a_{2}\right\rangle \cong\left\langle b_{1}, b_{2}\right\rangle$ means the forms have the same signature. And for dimension $n \geqslant 3$, the relation is defined recursively by 
$\left\langle a_{1}, a_{2}, \ldots, a_{n}\right\rangle \cong\left\langle b_{1}, b_{2}, \ldots, b_{n}\right\rangle$ if there exist $a, b, c_{3}, \ldots, c_{n} \in G$ such that $\left\langle a_{2}, \ldots, a_{n}\right\rangle$ $\cong\left\langle a, c_{3}, \ldots, c_{n}\right\rangle,\left\langle a_{1}, a\right\rangle \cong\left\langle b_{1}, b\right\rangle$ and $\left\langle b_{2}, \ldots, b_{n}\right\rangle \cong\left\langle b, c_{3}, \ldots, c_{n}\right\rangle$.

To see that the definition we have given for an abstract space of semiorderings is indeed the correct definition, we check that it has the main property of semiorderings for fields and semilocal rings: If we begin with a space of orderings $(X, G)$ and form the space of semiorderings $(Y, G)$, then the elements of $Y$ can be naturally identified with the group homomorphisms of the Witt ring $W(X, G)$ to $\mathbb{Z}$. The details for commutative rings and fields can be found in the work of Kleinstein and Rosenberg [17] and Knebusch [19]; for hermitian forms over certain *-fields, this is found in [10]. All of this will be subsumed under the present abstract approach. The fact that $X$ can be identified with the ring homomorphisms of $W(X, G)$ to $\mathbb{Z}$ is the content of [23, Theorem 2.3.3].

THEOREM 1.5. The set $Y$ can be naturally identified with the set of all group homomorphisms of $W(X, G)$ to $\mathbb{Z}$ which carry $\langle 1\rangle \mapsto 1$.

Proof: We need to show that any $y \in Y$ induces a well-defined mapping from $W(X, G)$ to $\mathbb{Z}$. From the definition of $W(X, G)$, we see that it suffices to consider forms $\varphi \cong \psi$ of dimension two, as dimension one is immediate and higher dimensions follow by induction. But in dimension two, $\varphi(x)=\psi(x)$ for all $x \in X$ by definition. Let $\varphi=\langle a, b\rangle$ and $\psi=\langle c, d\rangle$. We have $a(x)+b(x)=c(x)+d(x)$ for all $x \in X$ and $D\langle a, b\rangle=D\langle c, d\rangle$. For any $y \in Y$, if $a, b$ have the same sign, then that sign is shared by $c, d$ since the value sets are the same. If $a, b$ have opposite signs, then by the same argument, $c, d$ must also have opposite signs, so $a(y)+b(y)=c(y)+d(y)=0$ in this case. It follows that for any $y \in Y, a(y)+b(y)=c(y)+d(y)$ as desired.

Let $f: W(X, G) \rightarrow \mathbb{Z}$ be a group homomorphism taking $\langle 1\rangle \mapsto 1$. Elements of $G$ give rise to units in $W(X, G)$, so map to \pm 1 in $\mathbb{Z}$. Define $y \in\{-1,1\}^{G}$ by $y(g)=f(\langle g\rangle)$ for each $g \in G$. Then $y(-1)=-1$. If $h \in D\left\langle g_{1}, g_{2}\right\rangle$ and $y\left(g_{1}\right)=y\left(g_{2}\right)=1$, then $y(h)=f(h)=1$. By (1.1), we obtain $y \in Y$. Since $W(X, G)$ is generated as an additive group by one-dimensional forms and $f(\langle g\rangle)=y(g)$ for all $g \in G$, we see that $f$ coincides with the group homomorphism $\varphi \mapsto \varphi(y)$ determined by $y \in Y$.

A space of orderings $(X, G)$ is said to satisfy the strong approximation property $(S A P)$ if every clopen subset of $X$ can be written as $H(g)$ for some $g \in G$. For a formally real field $F$, we know that there is always a space of semiorderings, and it is strictly larger than $X$ if and only if $F$ does not satisfy SAP [25, Theorem 9.1]. This is generalised to semilocal rings by Kleinstein and Rosenberg in [17, Section 3]. However, [17, Section 1] actually goes further, with results which apply directly to the abstract spaces of orderings of Marshall and to our work here. Indeed, they work in slightly more generality than we have here. They say that $W(X, G)$ satisfies the Hasse-Minkowski principle $(H M P)$ if for every form $\varphi$ in $W(X, G)$, there exists an element $x \in X$ with $\varphi(x)=\min \{\operatorname{dim} \psi \mid \psi \sim \varphi\}$. From [17, pp. 881-882] we obtain

THEOREM 1.6. Let $(X, G)$ be a space of orderings. The following are equivalent: 
(1) $W(X, G)$ satisfies HMP.

(2) $(X, G)$ satisfies SAP.

(3) Every semiordering $y$ defined by condition (1.1) of Definition 1.2 actually lies in $X$.

\section{ApPlication to *-FIELDS: Semiorderings without ORDERINGS}

Let $D$ be a skew field with an involution $*$; let $S(D)=\left\{d \in D \mid d^{*}=d\right\}$ be the set of symmetric elements. Write $\Sigma(D)$ for the set of all sums of products of norms and elements of the commutator group $\left[D^{\times}, S(D)^{\times}\right]$. This replaces sums of squares in the field case with identity involution and is a multiplicative group if $0 \notin \Sigma(D)$. The work in this paper (which assumes, as in the definitions of Section 1, that there exists a space of orderings inside the space $Y$ ) applies to all *-fields in which $0 \notin \Sigma(D)$. When $0 \in \Sigma(D)$, there can be no *-orderings, but there may still be a more general subset called a Baer ordering, which is just a semiordering if $*$ is the identity.

Definition 2.1: A Baer ordering on $(D, *)$ is a subset $Q \subseteq S(D)^{\times}$satisfying $Q+Q \subseteq Q, d Q d^{*} \subseteq Q$ for all $d \in D^{\times}, 1 \in Q$, and $Q \cup-Q=S(D)^{\times}$.

The set of equivalence classes of anisotropic hermitian forms, $W(D, *)$, now loses its multiplicative structure and is only an additive group. However, the set $Y$ of Baer orderings can still be topologised as before and is still in bijective correspondence with group homomorphisms as in Theorem 1.5 .

As an example, consider $D=\mathbb{R}((x))((y))$ where $x y=-y x$ and the involution is induced by $x^{*}=x, y^{*}=y$ (see [9, Example 5.3] for more details). Then $(x y)^{*}=y^{*} x^{*}$ $=y x=-x y$ is a skew element. Since $-1=x y x^{*} y^{*} \in \Sigma(D)$, there are no *-orderings, but there are four Baer orderings determined by the signs of $x$ and $y$. Also, the fact that the skew element $x y$ cannot be in any Baer ordering means that the Harrison subbasic sets consist only of

$$
Y=H(1), \emptyset=H(-1), H(x), H(-x), H(y), H(-y),
$$

but this is not a group under symmetric difference as is normally the case, suggesting that there is no group in this situation to take the place of $G$ in the space of semiorderings $(Y, G)$. A solution to this is proposed in [13]. An analog to the Witt ring is defined by using the ring $W S(D, *)$ in $\mathrm{e}\left(Y_{D}, Z\right)$ generated by the image of the Witt group $W(D, *)$. In abstract terms, this means using the group generated by the Harrison subbasic elements. The content of [13, Theorem 2.4, Proposition 2.5] is that there are natural bijective correspondences between the Baer orderings, the ring homomorphisms $W S(D, *) \rightarrow \mathbb{Z}$, and the group homomorphisms $W(D, *) \rightarrow \mathbb{Z}$ carrying hermitian forms $\langle a\rangle$ into $\{ \pm 1\}$ for nonzero symmetric elements $a$, with $\langle 1\rangle \mapsto 1$. 
This allows us to view an abstract space of semiorderings as a prespace of orderings [1], with the group being the group of units in $W S(D, *)$. This gives rise to the most general class of rings considered in $[\mathbf{1 7}]$; in particular, this provides a general structure theory for these rings. With regard to Section 1 , we have that $Y$ is the space of minimal prime ideals of $W S(F)$, in the case where $D$ is a field $F$ with identity involution. Unfortunately, nothing is known about whether any additional structure applies beyond this extremely general setting for $W S(D, *)$. If valuations happen to behave nicely, there is some additional structure that occurs. This will be used in the main theorem below. The core idea is that we can compute exactly what happens to the spaces of semiorderings when one does a group extension of the space of orderings as in Theorem 3.2 below. Furthermore, this construction does not depend on the existence of a space of orderings inside the space of semiorderings. This is shown in [13, Theorem 2.8]. Instead of a group extension, one obtains a tensor product of the ring $W S\left(D_{v}, *\right)$ for the residue field with itself several times before doing a group extension. We give the details here in abstract form because they provide an alternate way of looking at the extension of semiorderings from $\bar{Y}$ to $Y$ which occurs in Theorem 3.2.

Let $G$ be a group of exponent 2 and let $\widehat{G}=\operatorname{Hom}(G,\{ \pm 1\})$ be the topological dual group of $G$ for the discrete topology on $G$. Let $-1 \neq 1$ be a distinguished element of $G$ and let $Y$ be a subset of $\widehat{G}$. The pair $(Y, G)$ is called a prespace of orderings if the following conditions hold:

$\mathrm{O}_{1} \quad Y$ is closed in $\widehat{G}$.

$\mathrm{O}_{2} \quad \sigma(-1)=-1$ for all $\sigma \in Y$.

$\mathrm{O}_{3}$ The element $g=1$ in $G$ is the unique element of $G$ such that $\sigma(g)=+1$ for all $\sigma \in Y$.

There are two fundamental constructions needed in dealing with spaces of orderings as well as a third one for the spaces of semiorderings mentioned above. We shall need all three and will describe them now. The first two can be found in [22] for spaces of orderings and in [1, Chapter IV, Section 2] for prespaces of orderings. The third construction, product, was introduced in [13]. The first two, particularly group extension, will be essential to the work of later sections.

DEFINITION 2.2: A prespace of orderings $(Y, G)$ is said to be a group extension of a prespace of orderings $(\bar{Y}, \bar{G})$, by a group $H$ of exponent 2 , if $(Y, G)=(\bar{Y} \times \chi(H), \bar{G} \times H)$ with distinguished element $(-1,1) \in G$ and action

$$
(\bar{y}, \sigma)(\bar{g}, h)=\bar{y}(\bar{g}) \sigma(h) \quad \text { for all }(\bar{y}, \sigma) \in \bar{Y} \times \chi(H) \text { and }(\bar{g}, h) \in \bar{G} \times H .
$$

Equivalently, we say that $(\bar{Y}, \bar{G})$ is the residue space of $(Y, G)$ with respect to $H$.

Thus $\bar{Y}$ is a quotient space of $Y$. We can recognise group extensions in abstract spaces of orderings or prespaces of orderings. For any prespace of orderings $(Y, G)$, we 
define the translation group

$$
\operatorname{Tr}(Y, G)=\{t \in \chi(G) \mid t Y=Y\} .
$$

Write

$$
\widetilde{G}=\{g \in G \mid t(g)=1 \forall t \in \operatorname{Tr}(Y, G)\} .
$$

It can be shown that $\operatorname{Tr}(Y, G)$ consists of all characters on $G$ which are trivial on $\tilde{G}$. Let $\widetilde{Y}$ be the image of $Y$ under the canonical restriction mapping $Y \rightarrow \chi(G) \rightarrow \chi(\widetilde{G})$. Choose any subgroup $H$ of $G$ such that $G=\widetilde{G} \times H$. Then $(Y, G)$ is a group extension of $(\widetilde{Y}, \widetilde{G})$ by $H \cong G / \widetilde{G}$. More generally, if $K$ is any closed subgroup of $\operatorname{Tr}(Y, G)$, let $\bar{G}=K^{\perp} \subseteq G$ and let $\bar{Y}$ be the image of $Y$ in $\chi(\bar{G})$ under restriction. Then $(\bar{Y}, \bar{G})$ is the residue space of $(Y, G)$ with respect to $K$.

DEFINITION 2.3: The sum of two prespaces of orderings $\left(Y_{1}, G_{1}\right)$ and $\left(Y_{2}, G_{2}\right)$ is the prespace of orderings $(Y, G)=\left(Y_{1} \cup Y_{2}, G_{1} \times G_{2}\right)$ with distinguished element $(-1,-1) \in G$. The action is defined by $\sigma_{i}\left(g_{1}, g_{2}\right)=\sigma_{i}\left(g_{i}\right)$ for all $\sigma_{i} \in X_{i}, i=1,2$, and $\left(g_{1}, g_{2}\right) \in G_{1} \times G_{2}$.

DeFinition 2.4: The product of two prespaces of orderings $\left(Y_{1}, G_{1}\right)$ and $\left(Y_{2}, G_{2}\right)$ is defined to be the prespace of orderings $(Y, G)=\left(Y_{1} \times Y_{2}, G_{1}+G_{2}\right)$, where $G_{1}+G_{2}$ is the coproduct in the category of elementary 2-groups with distinguished subgroup $\{ \pm 1\}$ preserved by all homomorphisms; equivalently,

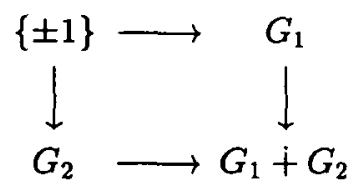

is a pushout diagram for homomorphisms preserving the distinguished element -1 . Constructively, $G_{1}+G_{2}$ is just $\left(G_{1} \times G_{2}\right) /\{(1,1),(-1,-1)\}$ so that $(1,-1)=(-1,1)$ is the distinguished element in $G_{1}+G_{2}$ and the action is given by $\left(\sigma_{1}, \sigma_{2}\right)\left(g_{1}, g_{2}\right)=\sigma_{1}\left(g_{1}\right) \sigma_{2}\left(g_{2}\right)$, for $\sigma_{i} \in Y_{i}$ and $g_{i} \in G_{i}, i=1,2$.

We shall say that a prespace of orderings $(Y, G)$ is Baer realisable if there exists a *field $(D, *)$ (possibly with $*$ the identity so that $D$ is commutative) such that $(Y, G)$ is the space of Baer orderings (semiorderings in the * = identity case), where $G$ is interpreted as above if $D$ is not commutative.

THEOREM 2.5. Let $(Y, G)$ be a Baer realisable prespace of orderings. Then the prespace of orderings $\left(Y^{\prime}, G^{\prime}\right)$ is also Baer realisable, where $\left(Y^{\prime}, G^{\prime}\right)$ is obtained by taking the product of $(Y, G)$ with itself, followed by a group extension by a group of order 2 .

Proof: Let $(D, *)$ be the $*$-field realising $(Y, G)$. Let $F$ be the field of central symmetric elements in $D$ (which equals $D$ if $*$ is the identity). Extend $F$ by forming the field of formal Laurent series $F((t))$ and let $D^{\prime}=D \otimes F((t))$, which is again a skew field [5, Section 2.3]. Extend * so that the central symmetric elements of $D^{\prime}$ become $F((t))$ 
(that is, by setting $t^{*}=t$ ). Let $v$ be the $t$-adic valuation on $D^{\prime}$. Since $t$ is symmetric, it is clear that $v(d)=v\left(d^{*}\right)$ for every $d \in D^{\prime}$; and since $t$ is central, it is clear that the smooth condition for a valuation (see [13, p. 93]) is satisfied. The value group $\Gamma$ is infinite cyclic, generated by $v(t)$, so $\Gamma / 2 \Gamma=\mathbb{Z}_{2}$. Finally, we need to know that every Baer ordering of $D^{\prime}$ is compatible with $v$ in the sense that $0<a \leqslant b$ implies $v(a) \geqslant v(b)$, for $a, b$ symmetric elements of $D^{\prime}$. One way to see this is to note that any element of $Y$ can extend to $D^{\prime}$ in at most two ways, determined by the sign of $t$, and all of these do occur and are compatible with $v$ by [9, Theorem 3.4]. An application of $[\mathbf{1 3}$, Theorem $2.8]$ now completes the proof.

For detailed skew field examples of the preceding theorem, see [13, Section 4]. At this point very little is known about which prespaces of orderings are Baer realisable. The example from [9] which begins this section is a space of Baer orderings, so of course is Baer realisable. But it is not a space of semiorderings as in Section 1 since it is a four point space which is not SAP. Indeed, the smallest space of orderings which is not SAP has four elements and it generates an 8 point space of semiorderings (as will be seen in the next section in Theorem 3.2). The example is in fact, a space of orderings in the abstract sense. There is no known example of a space of Baer orderings which is not either a space of orderings or a space of semiorderings associated with a space of orderings.

\section{GROUP EXTENSIONS AND MORPHISMS FOR SPACES OF ORDERINGS}

In this section we look at theorems which are motivated by theorems for real valuations on fields. The role of the value group will be taken by certain subgroups of $G$, and so is actually analogous to the value group modulo 2 for fields (and a slightly more complicated group for $*$-fields $[\mathbf{1 0}, \mathbf{1 1}]$ ). Our first result shows how to construct new spaces of semiorderings from old ones. The second result is analogous to the very general theorem that any valuation on a field can be lifted to any extension field.

We first show that Prestel's lifting theorems for semiorderings compatible with real valuations [25, Chapter 7] can be generalised to this setting. We replace valuations with group extensions of spaces of orderings, so we begin by describing the work of Marshall on these constructions.

Following $[23$, p. 62], we point out the connection between value sets for group extensions:

LEMma 3.1. Given $(X, G)$ a group extension of $(\bar{X}, \bar{G})$, let $s$ be an embedding of $G / \bar{G}$ into $G$ splitting the projection $G \rightarrow G / \bar{G}$, with $H=s(G / \bar{G})$. We then view $G=\bar{G} H$ and write elements of $G$ as $g=\bar{g} h$ or $g_{i}=\bar{g}_{i} h_{i}$, as needed. For any anisotropic 
form $\left\langle g_{1}, g_{2}\right\rangle$,

$$
D\left\langle g_{1}, g_{2}\right\rangle= \begin{cases}\left\{g_{1}, g_{2}\right\}, & \text { if } h_{1} \neq h_{2} \\ D\left\langle\bar{g}_{1}, \bar{g}_{2}\right\rangle \cdot h, & \text { if } h_{1}=h_{2}=h,\end{cases}
$$

where the value set $D\left\langle\bar{g}_{1}, \bar{g}_{2}\right\rangle$ is contained in $\bar{G}$.

Proof: The initial statement is a special case of $[23$, p. 62, Claim 2]. To obtain the final part, we claim that $D\left\langle g_{1}, g_{2}\right\rangle \subseteq \bar{G}$ when $h_{1}=h_{2}=1$ and $\left\langle\bar{g}_{1}, \bar{g}_{2}\right\rangle$ is anisotropic. This can be seen as follows: As a set of characters on $G$, we have $X=\bar{X} \times \chi(H)$ [23, p. 61]. Let $g=\bar{g} h \in D\left\langle g_{1}, g_{2}\right\rangle$. Since $h_{1}=h_{2}=1$, the elements $g_{1}$ and $g_{2}$ are constant on fibres over each element of $\bar{X}$. Since $\left\langle\bar{g}_{1}, \bar{g}_{2}\right\rangle$ is anisotropic, there exists some $\bar{x} \in \bar{X}$ at which they have the same sign; without loss of generality, assume $\bar{g}_{1}(\bar{x})=\bar{g}_{2}(\bar{x})=1$. Then $g$ must be 1 on the fibre over $\bar{x}$. That is, for any element $(\bar{x}, \chi) \in X$, we have $1=\bar{g}(\bar{x}) h(\chi)$. It follows that $h$ is constant when viewed as a function on $\chi(H)$, hence $h=1$ and $g=\bar{g} \in \bar{G}$.

THEOREM 3.2. Let $(X, G)$ be a group extension of $(\bar{X}, \bar{G})$, and $\bar{Y}$ the space of semiorderings for $(\bar{X}, \bar{G})$. Write $G=\bar{G} H$ as in the lemma. The space of semiorderings for $(X, G)$ satisfies

$$
Y \cong\{\sigma: H \rightarrow\{ \pm 1\} \mid \sigma(1)=1\} \times\{\mathfrak{P}: H \rightarrow \bar{Y}\},
$$

where the correspondence is given as follows: Given mappings $\sigma, \mathfrak{P}$ as above, define $y$ by

$$
y(g)=\mathfrak{P}(h)[\bar{g}] \cdot \sigma(h), \forall g=\bar{g} h \in G .
$$

Given a semiordering $y$, define $\sigma_{y}$ and $\mathfrak{P}_{y}$ via

$$
\sigma_{y}(h)=y(h) \quad \text { and } \quad \mathfrak{P}_{y}(h)[\bar{g}]=y(\bar{g} h) \sigma_{y}(h), \forall \bar{g} \in \bar{G}, h \in H .
$$

Proof: We first show that (1) defines an element of $(Y, G)$ : Note that $y \in\{-1,1\}^{G}$ and $y(-g)=-y(g)$, the latter from the fact that it holds for the semiordering $\mathfrak{P}(h)$. To show that $y \in Y$, we use condition (1.1) of Definition 1.2. Let $y\left(g_{1}\right)=y\left(g_{2}\right)=1$ and $g_{3} \in D\left\langle g_{1}, g_{2}\right\rangle$. To show $y\left(g_{3}\right)=1$, we follow the two cases of the previous lemma. First assume that $h_{1} \neq h_{2}$. Then without loss of generality, we may assume that $g_{3}=g_{1}$, from which the claim follows. Now assume that $h_{1}=h_{2}=h$. By Lemma 3.1, we obtain $h_{3}=h$ and $g_{3} \in D\left\langle\bar{g}_{1}, \bar{g}_{2}\right\rangle h$ so that $g_{3} \sigma(h) \in D\left\langle\bar{g}_{1} \sigma(h), \bar{g}_{2} \sigma(h)\right\rangle$. Since $y\left(g_{1}\right)=y\left(g_{2}\right)=1$, we have $\mathfrak{P}(h)$ is 1 at both $\bar{g}_{1} \sigma(h)$ and $\bar{g}_{2} \sigma(h)$. It follows that $y\left(g_{3}\right)=\mathfrak{P}(h)\left[\bar{g}_{3}\right] \sigma(h)=1$.

Secondly, let $\bar{y}=\mathfrak{P}_{y}(h)$. We show that $\bar{y} \in \bar{Y}$. Note that $\bar{y} \in\{ \pm 1\}^{\bar{G}}$ and that $\bar{y}(-1)$ $=-y(h) \sigma_{y}(h)=-1$. Let $\bar{g}_{1}, \bar{g}_{2} \in \bar{G}$ such that $\bar{y}\left(\bar{g}_{1}\right)=\bar{y}\left(\bar{g}_{2}\right)=1$; let $\bar{g}_{3} \in D\left\langle\bar{g}_{1}, \bar{g}_{2}\right\rangle$. If we can show that $\bar{y}\left(\bar{g}_{3}\right)=1$, we shall obtain $\bar{y} \in \bar{Y}$ by (1.1). Multiplying by $h$, we obtain $\bar{g}_{3} h \in D\left\langle\bar{g}_{1} h, \bar{g}_{2} h\right\rangle$. Now $\bar{y}\left(\bar{g}_{3}\right)=y\left(\bar{g}_{3} h\right) \sigma_{y}(h)$, which must agree with either $y\left(\bar{g}_{1} h\right) \sigma_{y}(h)=\bar{y}\left(\bar{g}_{1}\right)$ or $y\left(\bar{g}_{2} h\right) \sigma_{y}(h)=\bar{y}\left(\bar{g}_{2}\right)$, both of which equal 1 . 
Finally we must show that (1) and (2) give a bijective correspondence. For this, we look at the two compositions of the mappings. Given $y \in Y$, form $\mathfrak{P}_{y}$ and $\sigma_{y}$ by (2), and use them to define $y_{0}$ by (1). Then

$$
y_{0}(g)=\mathfrak{P}_{y}(h)[\bar{g}] \cdot \sigma_{y}(h)=y(\bar{g} h) \sigma_{y}(h) \cdot \sigma_{y}(h)=y(\bar{g} h)=y(g) .
$$

On the other hand, if we begin with a pair $(\sigma, \mathfrak{P})$, form $y$ by $(1)$ and then compute $\sigma_{y}$ and $\mathfrak{P}_{y}$, we obtain

$$
\begin{gathered}
\sigma_{y}(h)=y(h)=\mathfrak{P}(h)[1] \sigma(h)=\sigma(h) \quad \text { and } \\
\mathfrak{P}_{y}(h)[\bar{g}]=y(\bar{g} h) \sigma_{y}(h)=\mathfrak{P}(h)[\bar{g}] \sigma(h) \sigma_{y}(h)=\mathfrak{P}(h)[\bar{g}],
\end{gathered}
$$

for all $g=\bar{g} h \in G$.

This theorem, together with direct sum gives all the finitely generated spaces of semiorderings, when viewed as extensions of spaces of orderings. The product construction for prespaces of orderings presented in Section 2 is not explicitly needed as it is obtained from this theorem. The fact that all finitely generated spaces of orderings can be obtained beginning with a one point space and using group extension and direct sum was first proved for fields in [7] and then put in an abstract context by Marshall (see [22]). It is very useful to know when the elements of $Y$ are actually orderings, and this is the content of the next theorem. Again, this is based on Prestel's work for valuations [25, Theorem 7.9].

THEOREM 3.3. With the notation of Theorem 3.2, an element $y \in Y$ actually lies in $X$ if and only if $\mathfrak{P}_{y}: H \rightarrow \bar{Y}$ is a constant mapping onto an element of $\bar{X}$ and $\sigma_{y}: H \rightarrow\{ \pm 1\}$ is a character.

Proof: Assume first that $y \in X$. Then

$$
\sigma_{y}\left(h_{1} h_{2}\right)=y\left(h_{1} h_{2}\right)=\left(h_{1} h_{2}\right)(y)=h_{1}(y) h_{2}(y)=y\left(h_{1}\right) y\left(h_{2}\right)=\sigma_{y}\left(h_{1}\right) \sigma_{y}\left(h_{2}\right),
$$

so $\sigma_{y}$ is a character. Also,

$$
\mathfrak{P}_{y}(h)[\bar{g}]=y(\bar{g} h) \sigma_{y}(h)=y(\bar{g})\left(y(h) \sigma_{y}(h)\right)=y(\bar{g}),
$$

and thus $\mathfrak{P}_{y}$ is a constant mapping. Furthermore, since $\mathfrak{P}_{y}(1)[\bar{g}]=y(\bar{g})$ for all $\bar{g} \in \bar{G}$ and $y \in X$, it follows that $\mathfrak{P}_{y}(1)$ lies in $\bar{X}$.

Conversely, assume that $\sigma_{y}$ is a character and $\mathfrak{P}_{y}$ is a constant mapping onto an element of $\bar{X}$. We must show that $y$ preserves products, so that it is an element of the character group of $G$. Writing $g_{i}=h_{i} \bar{g}_{i}, i=1,2$, we have

$$
\begin{aligned}
y\left(g_{1} g_{2}\right) & =\mathfrak{P}_{y}\left(h_{1} h_{2}\right)\left[\bar{g}_{1} \bar{g}_{2}\right] \sigma_{y}\left(h_{1} h_{2}\right) \\
& =\mathfrak{P}_{y}\left(h_{1} h_{2}\right)\left[\bar{g}_{1}\right] \mathfrak{P}_{y}\left(h_{1} h_{2}\right)\left[\bar{g}_{2}\right] \sigma_{y}\left(h_{1}\right) \sigma_{y}\left(h_{2}\right) \\
& =\mathfrak{P}_{y}\left(h_{1}\right)\left[\bar{g}_{1}\right] \sigma_{y}\left(h_{1}\right) \mathfrak{P}_{y}\left(h_{2}\right)\left[\bar{g}_{2}\right] \sigma_{y}\left(h_{2}\right) \\
& =y\left(g_{1}\right) y\left(g_{2}\right),
\end{aligned}
$$


as desired.

Next we look at morphisms between spaces of orderings and how semiorderings and group extension constructions extend. In view of the preceding theorems, this gives an analog of the theorems for extending valuations on a field $F_{1}$ to an extension field $F_{2}$.

Recall that a morphism $\alpha:\left(X_{2}, G_{2}\right) \rightarrow\left(X_{1}, G_{1}\right)$ between two spaces of orderings is a mapping $\alpha: X_{2} \rightarrow X_{1}$ such that for each $g \in G_{1}$, the composite function $g \circ \alpha: X_{2}$ $\rightarrow\{-1,1\}$ is an element of $G_{2}$. In particular, $\alpha$ induces a group homomorphism $g \mapsto g \circ \alpha$ from $G_{1}$ to $G_{2}$ which we shall also denote by $\alpha$. Since $\alpha^{-1}(H(g))=H(g \circ \alpha)$ for each $g \in G_{1}$, such a mapping $\alpha$ is automatically continuous. There is a contravariant equivalence of categories between spaces of orderings and reduced Witt rings, where it is the mapping $G_{1} \rightarrow G_{2}$ that is the starting point, giving a Witt ring homomorphism $W\left(X_{1}, G_{1}\right) \rightarrow W\left(X_{2}, G_{2}\right)[22$, p. 140].

As we saw in Section 2 , using duality for the $\mathbb{Z} / 2 \mathbb{Z}$-vector space $\chi(G)$, we have a oneto-one correspondence between closed groups $\bar{G}$ with $\widetilde{G} \subseteq \bar{G} \subseteq G$ and closed subgroups $K$ of $\operatorname{Tr}(X, G)$ given by

$$
\begin{gathered}
K \longrightarrow G_{H}=\{g \in G \mid t(g)=1 \forall t \in K\} \quad \text { and } \\
\bar{G} \longrightarrow K_{\bar{G}}=\{t \in \operatorname{Tr}(X, G) \mid t(g)=1 \forall g \in \bar{G}\} .
\end{gathered}
$$

This gives us all the ways of viewing $(X, G)$ as a group extension, the smallest space of orderings "under" $(X, G)$ being $(\tilde{X}, \widetilde{G})$.

REMARK 3.4. While the construction giving the largest group extension always works in abstract spaces of orderings, it is not always reflected in valuations for fields. For example, if $F=\mathbb{Q}(\sqrt{2})((x))((y))$, the finest real valuation comes from the place of $F$ into $\mathbb{R}$ with residue field $\mathbb{Q}(\sqrt{2})$ and the value group modulo 2 has 4 elements. But the space of orderings is a fan with 8 orderings, so in the abstract one has a group $H$ of order 8 . The general situation is obtained by working with a subgroup of the translation group, and for this field example, that would be the elements of $\operatorname{Tr}(X, G)$ which fix the class of $\sqrt{2}$ modulo sums of squares.

LEMMA 3.5. Assume that the space of orderings $\left(X_{1}, G_{1}\right)$ is a group extension of $\left(\bar{X}_{1}, \bar{G}_{1}\right)$ and that $\alpha:\left(X_{2}, G_{2}\right) \rightarrow\left(X_{1}, G_{1}\right)$ is any morphism of spaces of orderings.

(1) The induced homomorphism $\alpha^{*}: \chi\left(G_{2}\right) \rightarrow \chi\left(G_{1}\right)$ carries the subgroup $\operatorname{Tr}\left(X_{2}, G_{2}\right)$ into the image of $\operatorname{Tr}\left(\alpha\left(X_{2}\right),\left.G_{1}\right|_{\alpha\left(X_{2}\right)}\right) \subseteq \chi\left(\left.G_{1}\right|_{\alpha\left(X_{2}\right)}\right)$ in $\chi\left(G_{1}\right)$. In particular, if $X_{2} \rightarrow X_{1}$ is surjective, then composing with $\alpha$ carries $\operatorname{Tr}\left(X_{2}, G_{2}\right)$ into $\operatorname{Tr}\left(X_{1}, G_{1}\right)$.

(2) Assume $\alpha: X_{2} \rightarrow X_{1}$ is surjective. Then $\left(X_{2}, G_{2}\right)$ is a group extension of a space $\left(\bar{X}_{2}, \bar{G}_{2}\right)$ and $\alpha$ induces a morphism from $\left(\bar{X}_{1}, \bar{G}_{1}\right)$ to $\left(\bar{X}_{2}, \bar{G}_{2}\right)$.

Proof: (1) Let $t_{2} \in \operatorname{Tr}\left(X_{2}, G_{2}\right)$ be arbitrary. Let $t_{1}=t_{2} \circ \alpha \in \chi\left(G_{1}\right)$. Since $t_{2} X_{2}=X_{2}$, we obtain $t_{1} X_{1}=X_{1}$ as follows. Given $x_{1} \in X_{1}$, lift it to $x_{2} \in X_{2}$; since 
$t_{2} x_{2} \in X_{2}$, the image $t_{1} x_{1}$ lies in $X_{1}$. Therefore, composing with $\alpha$ carries $\operatorname{Tr}\left(X_{2}, G_{2}\right)$ to $\operatorname{Tr}\left(X_{1}, G_{1}\right)$.

(2) Let $K_{1}$ be the subgroup of $\operatorname{Tr}\left(X_{1}, G_{1}\right)$ corresponding to $\bar{G}_{1}$ as above. Using (1), we obtain a subgroup $K_{2} \subseteq \operatorname{Tr}\left(X_{2}, G_{2}\right)$ as the inverse image of $K_{1}$. Let

$$
\bar{G}_{2}=\left\{g \in G_{2} \mid t(g)=1, \forall t \in K_{2}\right\}
$$

and

$$
\bar{X}_{2}=\operatorname{im}\left(X_{2} \hookrightarrow \chi\left(G_{2}\right) \rightarrow \chi\left(\bar{G}_{2}\right)\right) .
$$

Then $\left(\bar{X}_{2}, \bar{G}_{2}\right)$ is a space of orderings and $\left(X_{2}, G_{2}\right)$ is a group extension of it by a slight generalisation of [23, Theorem 4.1.3]. We need to define a morphism $\bar{\alpha}:\left(\bar{X}_{2}, \bar{G}_{2}\right)$ $\rightarrow\left(\bar{X}_{1}, \bar{G}_{1}\right)$, naturally induced by $\alpha$. We have a homomorphism $\bar{\alpha}: \bar{G}_{1} \hookrightarrow G_{1} \stackrel{\alpha}{\rightarrow} G_{2}$. We claim that the image lies in $\bar{G}_{2}$. Indeed, let $t_{2} \in K_{2}$, and $g_{2} \in \operatorname{im}(\bar{\alpha})$ with preimage $\bar{g}_{1} \in \bar{G}_{1}$. Let $t_{1}$ be the image of $t_{2}$ in $K_{1}$. Then $t_{2}\left(g_{2}\right)=t_{1}\left(g_{1}\right)=1$, so $g_{2} \in \bar{G}_{2}$. Thus we have a homomorphism $\bar{\alpha}: \bar{G}_{1} \rightarrow \bar{G}_{2}$. To obtain the corresponding mapping $\bar{X}_{2} \rightarrow \bar{X}_{1}$, note that we have a mapping $\bar{X}_{2} \hookrightarrow \chi\left(\bar{G}_{2}\right) \stackrel{\bar{\alpha}^{*}}{\rightarrow} \chi\left(\bar{G}_{1}\right)$. From the commutative diagram

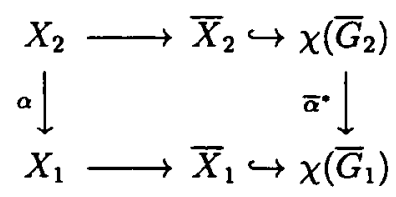

we see that $\bar{X}_{2}$ maps into $\bar{X}_{1}$ as desired.

THEOREM 3.6. Let $\alpha:\left(X_{2}, G_{2}\right) \rightarrow\left(X_{1}, G_{1}\right)$ be any morphism of spaces of orderings, and assume that the subspace $\left(X_{1}^{\prime}, G_{1}^{\prime}\right)$ of $\left(X_{1}, G_{1}\right)$ is a group extension of $\left(\bar{X}_{1}, \bar{G}_{1}\right)$. Let $X_{2}^{\prime}=\alpha^{-1}\left(X_{1}^{\prime}\right)$ and form the subspace $\left(X_{2}^{\prime}, G_{2}^{\prime}\right)$ of $\left(X_{2}, G_{2}\right)$ by setting $G_{2}^{\prime}=\left.G_{2}\right|_{X_{2}^{\prime}}$. Then $\left(X_{2}^{\prime}, G_{2}^{\prime}\right)$ is a group extension of a space $\left(\bar{X}_{2}, \bar{G}_{2}\right)$ and $\alpha$ induces a morphism from $\left(\bar{X}_{1}, \bar{G}_{1}\right)$ to $\left(\bar{X}_{2}, \bar{G}_{2}\right)$.

Proof: This is immediate from Lemma 3.5(2).

We next look at an abstract version of the old Artin-Schreier result on lifting an ordering from a field to an extension field.

THEOREM 3.7. Let $\left(Y_{2}, G_{2}\right) \stackrel{a}{\rightarrow}\left(Y_{1}, G_{1}\right)$ be a morphism of spaces of semiorderings. Then $y_{1} \in Y_{1}$ is in the image of $\alpha$ if and only if $-1 \notin D_{2}\left\langle\alpha\left(g_{1}\right), \ldots, \alpha\left(g_{n}\right)\right\rangle$ for any $g_{i} \in G_{1}$ such that $y_{1}\left(g_{i}\right)=1$ for all $i=1, \ldots, n$.

Proof: This requires the usual Zorn's lemma argument to construct $y_{2} \in Y_{2}$ extending $y_{1}$. By Zorn's lemma, there exists a maximal subset $A \subset G_{2}$ such that $\left\{\alpha\left(g_{1}\right), \ldots, \alpha\left(g_{n}\right)\right\} \subseteq A$ and $-1 \notin D\left\langle a_{1}, \ldots, a_{r}\right\rangle$ for all $r>0, a_{i} \in A$. First note that $A \cup-A=G_{2}$; otherwise, for $b \in G_{2}$, not in $A \cup-A$, we have $-1 \in D\left\langle b, a_{1}, \ldots, a_{r}\right\rangle$ $\cap D\left\langle-b, a_{1}, \ldots a_{r}\right\rangle$ for some $a_{i} \in A$, which is a contradiction since $b$ and $-b$ cannot both 
be negative. Define $y_{2}: G_{2} \rightarrow\{ \pm 1\}$ by

$$
y_{2}(g)=\left\{\begin{array}{ll}
+1, & \text { if } g \in A \\
-1, & \text { if } g \notin A
\end{array} .\right.
$$

For any $a, b \in A$ and $c \in D\langle a, b\rangle,-1 \in D\langle-c, a, b\rangle[23$, Theorem 2.2.6], so $-c \notin A$, then $c \in A$. It follows that $y_{2} \in Y_{2}$ by Definition 1.2.

\section{AbStract PREORDERINGS AND COVERING NUMBERS}

An abstract preordering is defined in [23, p. 33] as a proper subgroup $T$ of $G$ such that $a, b \in T \Longrightarrow D\langle a, b\rangle \subseteq T$. The set $T$ is the kernel of $\left.G \rightarrow G\right|_{S}$ for some subspace $S \subseteq X$ and conversely any subspace gives a preordering. This set $S$ will be denoted by

$$
X_{T}=\{x \in X \mid t(x)=1, \forall t \in T\} .
$$

Any subset $B \subseteq G$ generates a preordering, namely

$$
\left\{g \in G \mid g=1 \text { on } \bigcap_{b \in B} H(b)\right\} .
$$

A special type of preordering is a fan, which can be defined by requiring that if $x$ is any character of $G$ satisfying $x(-1)=-1$ and $x(t)=1$ for all $t \in T$, then $x \in X[\mathbf{2 3}$, Theorem 3.1.2]. This is equivalent to the associated Witt ring, $W\left(X_{T}, G / T\right)$, being an integral group ring. A few small examples are included in the chart in the appendix.

DEFINITION 4.1: For $S \subseteq Y$, the set

$$
T=\{g \in G \mid g \cdot \operatorname{ker} y \subseteq \operatorname{ker} y, \forall y \in S\}
$$

is the preordering covered by $S$. To see that it is indeed a preordering, observe that if $a, b \in T, c \in D\langle a, b\rangle$, then for any $h \in \operatorname{ker} y$, we have $c h \in D\langle a h, b h\rangle$ so $c h \in \operatorname{ker} y$. Conversely, given any preordering $T$, a cover of $T$ is a subset $S \subseteq Y$ for which $T$ is the preordering covered by $S$. A cover of $T$ always exists, since the space of semiorderings for the subspace $\left(X_{T}, T\right)$ of $(X, G)$, given by

$$
\{y \in Y \mid T \cdot \operatorname{ker} y \subseteq \operatorname{ker} y\},
$$

where $T \cdot \operatorname{ker} y=\{g h \mid g \in T, h \in \operatorname{ker} y\}$, is always a cover of $T$. There are typically a large number of different covers of $T$. For a given preordering $T$, the minimal cardinality for a cover $S$ is called the covering number of $T$. Covering numbers were introduced in [15] and studied further in [14]. 
For fields, if the covering number equals 1 , then there exists a nontrivial valuation fully compatible with the preordering. More generally, the covering number can be related to other invariants of a reduced Witt ring. Roughly speaking, it varies directly with the chain length and inversely with the stability index. A definition of chain length can be given in terms of the Harrison subbasis for an abstract space of orderings $(X, G)[\mathbf{2 3}$, Section 4.2]: $\operatorname{cl}(T)$ is the supremum of all integers $k$ for which there exists a chain

$$
H\left(a_{0}\right) \subsetneq H\left(a_{1}\right) \subsetneq \cdots \subsetneq H\left(a_{k}\right) \quad a_{k} \in G .
$$

The stability index is defined as the maximum integer $n$ such that there exists a fan $T \subseteq G$ with $\left|X_{T}\right|=2^{n}$ (or $\infty$ if no finite maximum exists) [23, p. 47]. The next theorem makes the connection between the invariants more precise. We shall return to the issue of invariants in Section 5

THEOREM 4.2. Let $X_{T}$ be a space of orderings of cardinality $n$. Let $r \leqslant \log _{2} n$ be the stability index.

(1) $\operatorname{cn}(T) \leqslant\left\{\begin{array}{ll}n, & \text { if } r=1 \\ n-2^{r}+1, & \text { if } r \neq 1\end{array}\right.$.

(2) $\operatorname{cn}(T) \leqslant \operatorname{cl}\left(X_{T}\right)$.

(3) The minimum values of covering number are

$$
\operatorname{cn}(T)= \begin{cases}1, & \text { if } n=1 \\ 2, & \text { if } n=2^{s}-2 \text { for some } s \geqslant 2 \\ 1, & \text { if } n \text { is even, not of the previous form } \\ 3, & \text { if } n=2^{s}-1 \text { for some } s \geqslant 2 \\ 2, & \text { if } n \text { is odd, not of the previous form }\end{cases}
$$

All bounds in this theorem are best possible.

Proof: We prove the theorem using recursion for reduced Witt rings of finite chain length. (1) and (2) are clear if $r=0\left(\left|X_{T}\right|=1\right)$ or $r=1\left(X_{T}\right.$ is SAP). In particular, the inequalities hold when $\left|X_{T}\right| \leqslant 3$. For a sum of spaces $X, X^{\prime}$ with stability indexes $r, r^{\prime}$, the covering numbers add by [14, Proposition 3.5], the chain length adds, and the stability index for the resulting space of orderings is $\max \left(r, r^{\prime}\right)$; thus (1) and (2) hold for the sum. If the formulas hold for $(\bar{X}, \bar{G})$ and $(X, G)$ is an extension of $(\bar{X}, \bar{G})$ by $\mathbb{Z}_{2}$, then the chain length is unchanged, the stability index increases by 1 and the covering number $c$ is halved if $c$ is even, and becomes $\{c / 2\rceil$ in general. Hence (2) is obvious and, assuming that $c \leqslant n-2^{r}+1$, we obtain

$$
\left\lceil\frac{c}{2}\right\rceil \leqslant c \leqslant n-2^{r}+1 \leqslant 2 n-2^{r+1}+1 .
$$


The fact that these bounds are best possible comes from the fact that for SAP spaces, covering number equals $|X|$ equals chain length, and for fans, both sides of (1) are equal to 1 since $n=2^{r}$.

(3) is clear for $n=1$ and $n=2$. Take a space of cardinality $n \geqslant 2$ and assume the formula holds for all smaller spaces. If $n$ is odd, the space must have the form of a sum of a one point space with a space of cardinality $n-1$, so we are done by the induction hypothesis in this case. Now assume that $n$ is even. Since covering numbers increase with sum and decrease with group extension, the minimum value must be achieved by taking a group extension of a space of cardinality $n / 2$, for which the covering number is minimal. The formula $\lceil c / 2\rceil$ for the resulting covering number, along with the induction hypothesis, now gives the claimed numbers for the minimum.

Note that this proof gives a construction for spaces with minimal covering number. Of particular interest are the numbers of the form $2^{s}-2,2^{s}-1, s=2,3,4, \ldots$ for which the minimum covering numbers are the largest. As seen in the proof, the spaces are created by alternately adding a point and forming a group extension.

We shall write $T[g]$ for the preordering generated by $T \cup\{g\}$; that is, the set

$$
\left\{h \in G \mid h=1 \text { on } X_{T} \cap H(g)\right\} .
$$

There is a preordering associated with any semiordering, namely the preordering covered by it. For a fixed semiordering $y \in Y$, we write

$$
T_{y}=\{g \in G \mid g \cdot \operatorname{ker} y \subseteq \operatorname{ker} y\} .
$$

This preordering, of course, has covering number one and such preorderings have a special role in quadratic form theory, as shown in [14].

We wish to see how to compute $T_{y}$, particularly in terms of Theorem 3.2. We assume that $(Y, G)$ is a space of semiorderings for $(X, G)$.

PROPOSITION 4.3. Let $y \in Y$ be a proper semiordering.

(2) Assume there exist $g_{1}, g_{2} \in G$ with $y\left(g_{1}\right)=y\left(g_{2}\right)=-y\left(g_{1} g_{2}\right)=1$. Then there exist four orderings $x_{i} \in X, i=1,2,3,4$, with

$$
T_{y} \subseteq T=\left\{g \in G \mid x_{i}(g)=1, i=1,2,3,4\right\}
$$

and such that the four orderings are distinguished by the signs of $g_{1}, g_{2}$. Furthermore $T$ is a fan.

(3) An ordering $x$ lies in $X_{T_{y}}$ if and only if for any $g_{1}$ with $-x\left(g_{1}\right)=y\left(g_{1}\right)=1$, there exists $g_{2} \in \operatorname{ker} y$ with $y\left(g_{1} g_{2}\right)=-1$.

PROof: (1) Since $g_{1},-g_{1} \notin T_{y}$, the set $T_{y}\left[g_{1}\right]$ is again a preordering. Furthermore, the conditions on $y$ imply that $g_{2},-g_{2} \notin T_{y}\left[g_{1}\right]$, so that $T_{y}\left[g_{1}, g_{2}\right]$ is also a preordering. 
By Zorn's lemma, there is an ordering $x_{1}$ positive on all elements of $T_{y}\left[g_{1}, g_{2}\right]$. Now apply Zorn's lemma again to obtain a maximal preordering $T$ such that $T_{y} \subseteq T \subseteq \operatorname{ker} x_{1}$ and $T$ does not contain any of $\pm g_{1}, \pm g_{2}, \pm g_{1} g_{2}$. Then $x_{1}$ and the other three orderings in $X_{T}$ clearly fulfill the conditions of the theorem.

$(2)(\Longrightarrow)$ Since $x\left(g_{1}\right)=-1$ implies that $g_{1} \notin T_{y}$, we know that $g_{1}$ ker $y \&$ ker $y$. That is, there exists $g_{2} \in \operatorname{ker} y$ such that $g_{1} g_{2} \notin \operatorname{ker} y$.

( ) Given $g_{1} \in T_{y}$, if $y\left(g_{1}\right)=1$ we are done. If not, then by hypothesis, there exists $g_{2} \in \operatorname{ker} y$ such that $y\left(g_{1} g_{2}\right)=-1$, contradicting the fact that $g_{1} \in T_{y}$.

Using this proposition, we can now give a computation of the orderings containing $T_{y}$ in terms of the representation of semiorderings found in Theorem 3.2. We require one further piece of terminology. Given a subset $X^{\prime}$ of $X$, the saturation of $X^{\prime}$ is defined to be

$$
X^{\prime}=\left\{x \in X \mid x(g)=1 \text { for all } g \in \bigcap_{x^{\prime} \in X^{\prime}} \operatorname{ker} x^{\prime}\right\}
$$

If $X^{\prime}$ is equal to its saturation, we say $X^{\prime}$ is saturated [17, Definition 1.7]. The need for this in the next theorem comes from the fact that if three orderings in $X^{\prime}$ are in $X_{T}$ for some fan $T$ with $\left|X_{T}\right|=4$, then the fourth ordering is in the saturation of $X^{\prime}$. Indeed, if $X^{\prime}$ is clopen, all additional elements of the saturation occur in this way [23, Theorem 4.3.4 and Note 4, p. 126].

THEOREM 4.4. Let $y \in Y$ correspond to the pair $(\sigma, \mathfrak{P})$ in Theorem 3.2. The orderings in $X_{T_{v}}$ are precisely those orderings given by pairs $\left(\sigma_{0}, \mathfrak{P}_{0}\right)$ satisfying the following condition:

$$
\begin{gathered}
\text { If } y(\bar{g} h)=1 \text { and } \mathfrak{P}_{0}(h)[\bar{g}]=-\sigma_{0}(h), \text { then there exist } \\
\bar{g}^{\prime} \in \bar{G} \text { and } h^{\prime} \in H, \text { such that } y\left(\bar{g}^{\prime} h^{\prime}\right)=1 \text { and } y\left(\overline{g g^{\prime}} h h^{\prime}\right)=-1 .
\end{gathered}
$$

Furthermore, we have

$$
T_{y} \cap \bar{G}=\bigcap_{h \in H} T_{\mathfrak{P}(h)}
$$

and $\mathfrak{P}_{0}$ is a constant mapping onto some ordering in the saturation of the set $\bigcup_{h \in H} \bar{X}_{T_{\mathfrak{P}(h)}}$. Moreover, given any such ordering $\bar{x}$ in $\bar{X}$, if we define $\mathfrak{P}_{0}$ to be the constant map onto $\bar{x}$, then there exists a character $\sigma_{0}: H \rightarrow\{ \pm 1\}$ such that the ordering $\left(\sigma_{0}, \mathfrak{P}_{0}\right)$ lies in $X_{T_{y}}$

Proof: The main part of the theorem is just a restatement of Proposition 4.3(2). To prove equation (4.2), first assume that $\bar{g} \in T_{y} \cap \bar{G}$. Let $h \in H, \mathfrak{P}(h)\left[\bar{g}^{\prime}\right]=1$. We must show that $\mathfrak{P}(h)[\overline{g g}]=1$. From Theorem 3.2 we have

$$
y\left(\bar{g}^{\prime} h\right)=\mathfrak{P}(h)\left[\bar{g}^{\prime}\right] \sigma(h)=\sigma(h) .
$$


And by definition of $T_{y}$, we have

$$
y\left(\bar{g}^{\prime} h\right)=y\left(\bar{g}^{\prime} h\right)=\mathfrak{P}(h)\left[\overrightarrow{g g}^{\prime}\right] \sigma(h),
$$

from which we obtain $\mathfrak{P}(h)\left[\bar{g} \bar{g}^{\prime}\right]=1$, hence $\bar{g} \in T_{\mathfrak{P}(h)}$. Conversely, assume that $\bar{g} \in \bigcap_{h \in H} T_{\mathfrak{P}(h)}$. Let $\bar{g}^{\prime} h^{\prime} \in \operatorname{ker} y$. Then

$$
y\left(\overline{g g^{\prime}} h^{\prime}\right)=\mathfrak{P}\left(h^{\prime}\right)[\overline{g g}] \sigma\left(h^{\prime}\right)=\mathfrak{P}\left(h^{\prime}\right)\left[\bar{g}^{\prime}\right] \sigma\left(h^{\prime}\right)=y\left(\bar{g}^{\prime} h^{\prime}\right)=1 .
$$

Since this works for all elements of $\operatorname{ker} y$, we have $\bar{g} \in T_{y}$.

Now consider any ordering $x \in T_{y}$, corresponding to the pair $\left(\sigma_{0}, \mathfrak{P}_{0}\right)$. Let $\bar{x}$ denote the constant value of $\mathfrak{P}_{0}$. Since $x$ is positive on $T_{y}$, condition (4.1) implies that $\bar{x}$ contains the preordering $\bigcap_{h \in H} T_{\mathfrak{P}(h)}$. This implies the conclusion by the definition of saturation.

Finally, given any $\bar{x}$ in this saturation, with $\mathfrak{P}_{0}: H \rightarrow \bar{X}$ defined as the constant mapping onto $\bar{x}$, we need to define a corresponding $\sigma_{0}$ satisfying $\sigma_{0}(h)=\mathfrak{P}_{0}(h)[\bar{g}]$, when $\bar{g} h \in T_{y}$; for $H^{\prime}$ equal to a complementary subgroup of $H$ to $\left\{h \in H \mid(\exists \bar{g} \in \bar{G}) \bar{g} h \in T_{y}\right\}$, choose a $\mathbb{Z} / \mathbb{Z}_{2}$-basis of $H^{\prime}$, define $\sigma_{0}$ arbitrarily on this basis and extend it by linearity. If $\sigma_{0}$ is well-defined (that is, independent of the choice of $\bar{g}$ ), we shall be done by (4.1). Assume that $\bar{g} h, \bar{g}^{\prime} h \in T_{y}$. Then the product

$$
\bar{g} h \bar{g}^{\prime} h=\overline{g g}^{\prime} \in T_{y} \cap \bar{G}=\bigcap_{h \in H} T_{\mathfrak{P}(h)} .
$$

By the choice of $\mathfrak{P}_{0}$, we have $\mathfrak{P}_{0}(h)\left[\overline{g g^{\prime}}\right]=1$; but since $\bar{x}=\mathfrak{P}_{0}(h)$ is an ordering, this equals $\mathfrak{P}_{0}(h)[\bar{g}] \mathfrak{P}_{0}(h)\left[\bar{g}^{\prime}\right]$, so that $\mathfrak{P}_{0}(h)[\bar{g}]=\mathfrak{P}_{0}(h)\left[\bar{g}^{\prime}\right]$, thus $\sigma_{0}$ is well-defined.

Condition (4.1) is little more than a complicated restatement of the fact that an ordering in $X_{T}$ contains $T$. But it does provide an interesting perspective. It could also be written as saying that if $\left(\sigma_{0}, \mathfrak{P}_{0}\right) \in X_{T_{y}}$, then for $g=\bar{g} h \in T_{y}$, we must have $\sigma_{0}(h)=\mathfrak{P}_{0}(h)[\bar{g}]$. By the general lifting result, Theorem 3.2, this condition on $\sigma_{0}$ is not needed if $X_{T_{y}}=X$, as all choices of the character $\sigma_{0}$ work. But this is not true in general as the next example shows. As in the previous proof, we see that $\sigma_{0}$ can be arbitrarily defined on basis elements for the subgroup of $H$ not involved in $T_{y}$ (that is, $h_{1}$ below, but not $h_{2}$ ). The example also shows the effect of the extension group not having a canonical embedding in $G$.

EXAMPLE 4.5. We work with the example from the appendix with $|X|=12$ and $|Y|$ $=648$. This can be viewed as a group extension by a group $H=\left\{1, h_{1}, h_{2}, h_{1} h_{2}\right\}$ of a three point space $\bar{X}=\bar{Y}=\left\{\bar{y}_{1}, \bar{y}_{2}, \bar{y}_{3}\right\}$, with group $\bar{G}=\left\{ \pm 1, \pm \bar{g}_{1}, \pm \bar{g}_{2}, \pm \bar{g}_{i} \bar{g}_{2}\right\}$, where we may choose $\bar{g}_{i}$ to be positive on $\bar{y}_{i}(i=1,2)$ and negative on the other two orderings. In this space $(X, \bar{G} H)$, we let $T$ be the fan $\left\{1,-\bar{g}_{1} h_{2},-\bar{g}_{1} \bar{g}_{2}, \bar{g}_{2} h_{2}\right\}$. One can easily check that $T$ is covered by the semiordering $y$ where

$$
\begin{aligned}
\operatorname{ker} y=\left\{1, \bar{g}_{1},-\bar{g}_{2},\right. & -\bar{g}_{1} \bar{g}_{2}, h_{1},-\bar{g}_{1} h_{1}, \bar{g}_{2} h_{1},-\bar{g}_{1} \bar{g}_{2} h_{1}, \\
& \left.-h_{2},-\bar{g}_{1} h_{2}, \bar{g}_{2} h_{2}, \bar{g}_{1} \bar{g}_{2} h_{2}, h_{1} h_{2},-\bar{g}_{1} h_{1} h_{2}, \bar{g}_{2} h_{1} h_{2},-\bar{g}_{1} \bar{g}_{2} h_{1} h_{2}\right\} .
\end{aligned}
$$


This semiordering is expressed in terms of Theorem 3.2 as

$$
\begin{gathered}
\mathfrak{P}: 1 \mapsto \bar{y}_{1}, h_{1} \mapsto \bar{y}_{2}, h_{2} \mapsto \bar{y}_{1}, h_{1} h_{2} \mapsto \bar{y}_{2} \\
\sigma: 1 \mapsto 1, h_{1} \mapsto 1, h_{2} \mapsto-1, h_{1} h_{2} \mapsto 1 .
\end{gathered}
$$

The four orderings associated with $T$ are given by

$$
\begin{array}{ll}
\mathfrak{P} \equiv \bar{y}_{1} ; & \sigma: h_{1} \mapsto 1, h_{2} \mapsto-1 \\
\mathfrak{P} \equiv \bar{y}_{1} ; & \sigma: h_{1} \mapsto-1, h_{2} \mapsto-1 \\
\mathfrak{P} \equiv \bar{y}_{2} ; & \sigma: h_{1} \mapsto 1, h_{2} \mapsto 1 \\
\mathfrak{P} \equiv \bar{y}_{2} ; & \sigma: h_{1} \mapsto-1, h_{2} \mapsto 1,
\end{array}
$$

where $\sigma$ is a character determined by its values on $h_{1}, h_{2}$, as required for an ordering.

There are always many different semiorderings that could be used to cover a preordering; in this case, there are four choices for $y$. A different choice (where $\bar{g}_{1}<0, \bar{g}_{2}>0$ ) will make $\sigma \equiv 1$. But there are fans where this choice of $\sigma$ cannot be obtained. The choice of $H$ is not canonical. If $H$ is replaced by $\left\{1, \bar{g}_{1} h_{1},-\bar{g}_{1} h_{2},-h_{1} h_{2}\right\}$, then $T$ can be written as $(T \cap \bar{G}) \times(T \cap H)$.

EXAMPLE 4.6. For a preordering with covering number 1, it would be nice to be able to find all of the semiorderings which cover it. In principle, Theorem 4.4 can be used for this, though it can be quite complicated for large spaces. There are cases where it is quite feasible, however, and we use the space of Example 4.5 to demonstrate this. We want to describe an arbitrary semiordering $(\sigma, \mathfrak{P})$ covering the space. By $(3.1)$, we view the space $Y$ as

$$
\{\sigma: H \rightarrow\{ \pm 1\} \mid \sigma(1)=1\} \times\{\mathfrak{P}: H \rightarrow \bar{Y}\}
$$

which has cardinality $2^{3} \cdot 3^{4}=648$ since $H \cong \mathbb{Z}_{2} \times \mathbb{Z}_{2}$. In this case, $\bar{X}=\bar{Y}$ is SAP, so all subsets are saturated and there are no proper semiorderings. It follows that the image of $\mathfrak{P}$ must be all of $\bar{X}$ since each $\bar{y}_{i} \in \bar{X}$ has liftings in $X_{T_{y}}=X$. There are 36 ways to map $H$ onto $\bar{X}$. In our case, there is no restriction on $\sigma$ and all 8 choices work; this is because there is no proper subspace with covering number 1 having a residue space with 3 elements. It follows that there are $8 \cdot 36=288$ semiorderings which cover the space, and we know exactly how to construct them.

For a fan, there is an easy way to write down a specific semiordering which covers it [21, Proposition 14.12]. We are able to extend that result to any preordering with covering number one.

LEMMA 4.7. Let $(Y, G)$ be a space of semiorderings associated with $(X, G)$. Let $y_{1}, y_{2} \in Y$. If there exists $g \in G$ such that $g \operatorname{ker} y_{1}=\operatorname{ker} y_{2}$, then $T_{y_{1}}=T_{y_{2}}$.

Proof: Assume that $g$ ker $y_{1}=\operatorname{ker} y_{2}$. This means that $g \operatorname{ker} y_{2}=g^{2} \operatorname{ker} y_{1}=\operatorname{ker} y_{1}$ also. Let $t \in T_{y_{1}}$, so that $t \operatorname{ker} y_{1}=\operatorname{ker} y_{1}$. For any $h \in \operatorname{ker} y_{1}$, we have

$$
t(g h)=g(t h) \in g \operatorname{ker} y_{1}=\operatorname{ker} y_{2} .
$$


Since $g h$ represents an arbitrary element of $\operatorname{ker} y_{2}$, we obtain $t \in \operatorname{ker} y_{2}$, so that $T_{y_{1}}=T_{y_{2}}$.

Let $T$ be a preordering with covering number one and $\left|X_{T}\right|>1$. Then $\left(X_{T}, G_{T}\right)$ must be a group extension of a space of orderings $(\bar{X}, \bar{G})$ by the group $H=\operatorname{Tr}\left(X_{T}, G_{T}\right)$ with $|H| \geqslant \operatorname{cn} \bar{X}$ by [14, Proposition 3.5]. We shall distinguish two cases, depending on $\operatorname{cn} \bar{X}$. If $\operatorname{cn} \bar{X}=1$, then actually $|\bar{X}|=1$, since otherwise $(\bar{X}, \bar{G})$ would be a proper group extension and $H$ would not actually equal $\operatorname{Tr}\left(X_{T}, G_{T}\right)$. The case where $|\bar{X}|=1$ is the known fan case mentioned above.

THEOREM 4.8. Let $T$ be a preordering with covering number one and $\left|X_{T}\right|>1$. Let $H$ and $(\bar{X}, \bar{G})$ be as above. Let $\mathbf{C}=\left\{y_{1}, y_{2}, y_{3}, \ldots\right\}$ be a minimal covering for $\bar{X}$.

(1) If $\operatorname{cn} \bar{X}=1$, let $x \in X$ and define $y: G \rightarrow\{ \pm 1\}$ by

$$
\operatorname{ker} y=\{1\} \cup\{-h \mid h \in H\} \text {. }
$$

(2) If $\mathrm{cn} \bar{X}>1$, for each $h \in H, h \neq 1$, assign an element $y_{h} \neq y_{1}$ in $\mathrm{C}$ so that all elements of $\mathbf{C}$ are used, including $y_{1}$ assigned to $1 \in H$. Define $y: G \rightarrow\{ \pm 1\}$ by

$$
\operatorname{ker} y=\operatorname{ker} y_{1} \cup \bigcup_{1 \neq h \in H}-h \operatorname{ker} y_{h} .
$$

Then $y$ is a semiordering covering $T$.

Proof: (1) For our abstract setting, $T=\{1\}$ and $X=\chi(H)$. Thus one choice for an ordering in $X$ is defined by

$$
\operatorname{ker} x=\{(1, h) \mid h \in H\} \subseteq\{ \pm 1\} \times H .
$$

With this notation, our expression in (4.3) is just a translation of [21, Proposition 14.12].

(2) We first check that $y$ is a semiordering. Notice first that the sets in the union defining $y$ are all disjoint since they lie in different cosets of $G_{T} / \bar{G}$. Thus the only part of condition (1.1) of the definition that is not obvious is closure under addition, and this follows from Lemma 3.1 and the fact that each $y_{h}$ is a semiordering.

A Zorn's lemma argument shows that the intersection of all kernels of semiorderings in $Y_{T}$ is precisely equal to $T$ (see, for example [25, Corollary 1.14]). From the definition of group extension, these elements must all lie in $\bar{G}$, and so we actually have

$$
T=\bigcap_{h \in H} T_{y_{h}} .
$$

Clearly $T \cdot \operatorname{ker} y \subseteq \operatorname{ker} y$. Finally, assume that $g \operatorname{ker} y \subseteq \operatorname{ker} y$. In particular, $g \in \operatorname{ker} y$, so lies in one of the subsets of the union. Assume first that $g \in \operatorname{ker} y_{1}$. Certainly, we must have $g \operatorname{ker} y_{1} \subseteq \operatorname{ker} y_{1}$, so $g \in T_{y_{1}}$. For any $h \neq 1, h \in H$, we must have

$$
g\left(-h \operatorname{ker} y_{h}\right)=-h\left(g \operatorname{ker} y_{h}\right) \subseteq-h \operatorname{ker} y_{h}
$$


which implies that $g$ ker $y_{h} \subseteq \operatorname{ker} y_{h}$; that is $g \in T_{y_{h}}$. By (4.5), we obtain $g \in T$. It remains to consider the case where $g=-h g_{h}$ for some $g_{h} \in \operatorname{ker} y_{h}$. Then $g \cdot \operatorname{ker} y_{1}$ $=-h \cdot\left(g_{h} \operatorname{ker} y_{1}\right) \subseteq \operatorname{ker} y$ implies that $g_{h} \operatorname{ker} y_{1}=\operatorname{ker} y_{h}$. But this contradicts Lemma 3.10 , as our choice of the semiorderings was such that they form a minimal covering of $\bar{X}$, and thus must have different associated preorderings.

\section{COMPUTATIONS FOR SMALL SPACES OF ORDERINGS}

Group extension together with direct sum gives all the finitely generated spaces of orderings, and hence of semiorderings using the construction of Definition 1.2. This allows one to do the combinatorial sort of work found in [8] and Merzel [24] for spaces of orderings. The tree structure described in [8] defines the finite spaces of semiorderings as well as spaces of orderings. In this section we take a brief look at the power of some of the new invariants obtained from semiorderings in characterising reduced Witt rings (or equivalently, spaces of orderings). The pair of invariants $|X|$ and $|Y|$ are surprisingly powerful for just two numbers in determining finitely generated rings $W(X, G)$.

Proposition 5.1. For $|X|<12$, the space of orderings $(X, G)$ is completely determined by the two numbers $|X|$ and $|Y|$. For $|X|=12$, of the 16 different rings, there are two with $|Y|=24$ and two with $|Y|=36$.

Proof: This is simply a matter of computation. The rings with $(|X|,|Y|)$ $=(12,24)$ are $\mathbb{Z}^{3}[w] \times \mathbb{Z}[x] \times \mathbb{Z}[y] \times \mathbb{Z}[z]$ and $(\mathbb{Z}[x, y])^{3}$ and the rings with $(|X|,|Y|)$ $=(12,36)$ are $\left(\mathbb{Z}^{3}[x]\right)^{2}$ and $\mathbb{Z}[v] \times \mathbb{Z}[w] \times(\mathbb{Z}[x] \times \mathbb{Z}[y])[z]$.

At 18 orderings, one first finds two Witt rings with the same $|X|,|Y|,|G|$ and stability index but different covering number and chain length. The four examples of the Proposition are distinguished by covering numbers, which are $8,3,4$ and 6 , respectively. For $|X|<24$, the set of invariants $|X|,|Y|,|G|$, covering number, chain length, stability index and number of components completely determine the space of orderings. The power of $|Y|$ is seen with $|X|=24$, where there are seven spaces of orderings with $|G|=13$, covering number 5 , chain length 10 , stability index 3 , and having exactly 2 components. These are distinguished by $|Y|=256,260,272,292,320,356$ and 400 . There also exist two spaces with 24 orderings for which all seven invariants are identical: $|X|=24,|Y|=108$, $|G|=2^{15}, \mathrm{cn}=7, \mathrm{cl}=12$, stability index is 2 and having 3 components. These are the spaces with Witt rings

$$
\left(\mathbb{Z}^{5}[x]\right)^{2} \times \mathbb{Z}^{2}[y] \quad \text { and } \quad\left(\mathbb{Z}^{3}[x]\right)^{2} \times \mathbb{Z}^{6}[y]
$$

It is easy to make up artificial invariants to distinguish particular spaces of orderings, but there is little one can do in general. Notice that all of the standard invariants mentioned above, with the exceptions of stability index and $|G|$, have the property that for sums of spaces of orderings, they add the corresponding invariants. This limits their ability to 
distinguish spaces of orderings. Furthermore, stability index is a very weak invariant and $|G|$ can be replaced by $\log _{2}|G|$ which is additive.

THEOREM 5.2. No finite collection of rational invariants which are additive over sums of spaces of orderings can distinguish all spaces of orderings.

Proof: Assume there are $n$ invariants. Without loss of generality, we may assume that they are not all zero on the one point space. For the purposes of this proof, let $F_{j}$ denote a fan with $2^{j}$ orderings. Let $A_{i j}$ be the value of the $i$ th invariant on the fan $F_{j}, j=1,2, \ldots$ We construct two spaces with all $n$ invariants identical. As our first approximation to the construction, consider a space which is a sum of $F_{n+1}$ and some (to be determined) number of copies of $F_{1}$. For the other space, we take sums of copies of $F_{j}, j=2, \ldots n$. We will have our examples if we can solve the system of linear equations

$$
\left(\begin{array}{c}
A_{1, n+1} \\
\vdots \\
A_{n, n+1}
\end{array}\right)=\left(\begin{array}{cccc}
A_{1,2} & \ldots & A_{1, n} & -A_{1,1} \\
& \vdots & & \\
A_{n, 2} & \ldots & A_{n, n} & -A_{n, 1}
\end{array}\right)\left(\begin{array}{c}
x_{1} \\
\vdots \\
x_{n}
\end{array}\right)
$$

If the given matrix is nonsingular, that is, if the invariants are linearly independent for fans of stability index at most $n$, we obtain rational solutions. If the common denominator $m$ of those solutions is not one, we use $m$ copies of the fan $F_{n+1}$ and all of our solutions become integers. If any happen to be negative, the corresponding fans are moved to the other space of orderings. Now, if the invariants are dependent for fans of stability index up to and including $n+1$, we can just eliminate the dependent ones and use the argument above on the reduced list of invariants. We are left with the situation in which the $n$ invariants become independent only when we include $A_{k, n+1}, k=1, \ldots, n$. But then the matrix

$$
\left(\begin{array}{ccccc}
A_{1,2} & \ldots & A_{1, n} & A_{1, n+1} & -A_{1,1} \\
& \vdots & & & \\
A_{n, 2} & \ldots & A_{n, n} & A_{n, n+1} & -A_{n, 1}
\end{array}\right)
$$

has rank $n$ and one of the first $n-1$ columns can be eliminated to make the resulting square matrix nonsingular. In this case, our starting point for the two spaces of orderings will be the sum of the fan $F_{n+2}$ with $x_{n}$ two point spaces $F_{1}$ and the sum of the remaining fans determined by the remaining first $n-1$ columns in the resulting $n \times n$ matrix. Then modify the spaces as above for rational or negative solutions.

It may happen that one can construct integral invariants recursively that do distinguish all finite spaces of orderings. For example, they might be based on using products instead of sums for combining the invariants of the components. But these are not practical in that they tend to grow very rapidly. Something of a compromise is found in an invariant introduced in [12, Section 5]. For a formally real pythagorean field $F$, one can show that the space of orderings is determined by $\Phi\left(\mathcal{J}_{F}\right)$, the Frattini subgroup of an 
involution subgroup (see [12, Section 2]) of a certain Galois extension of $F$. Using Galois cohomology, one can compute the number

$$
\log _{2}\left|\Phi\left(\mathcal{J}_{F}\right)\right|=\left(\begin{array}{c}
m \\
2
\end{array}\right)-\operatorname{dim}_{\mathbb{Z}_{2}} H^{2}\left(\operatorname{Gal}(F(2) / F), \mathbb{Z}_{2}\right),
$$

where $m=\log _{2}\left|\dot{F} / \dot{F}^{2}\right|[12$, Theorem 5.5]. This is shown to be an invariant of the space of orderings, not depending on which field $F$ gives that space. We write $\varphi(X, G)$ for the value of $\log _{2}\left|\Phi\left(\mathcal{J}_{F}\right)\right|$, where $X=X_{F}$ and $G=\dot{F} / \dot{F}^{2}$. For our purposes here, all we need to know about the invariant is how to compute it recursively, and this follows from [12, Proposition 5.3].

\section{Proposition 5.3 .}

(1) If $X$ is the one point space, then $\varphi(X,\{ \pm 1\})=0$.

(2) If $(X, G)$ is the sum of two spaces of orderings $\left(X_{1}, G_{1}\right)$ and $\left(X_{2}, G_{2}\right)$, then

$$
\varphi(X, G)=\varphi\left(X_{1}, G_{1}\right)+\varphi\left(X_{2}, G_{2}\right)+\left(\log _{2}\left|G_{1}\right|\right)\left(\log _{2}\left|G_{2}\right|\right) .
$$

(3) If $(X, G)$ is a group extension of $(\bar{X}, \bar{G})$ by $\mathbb{Z}_{2}$, then

$$
\varphi(X, G)=\varphi(\bar{X}, \bar{G})+1 .
$$

Even though this invariant is independent of the other invariants above, it has the problem that it does not do well at distinguishing spaces of low stability index. Indeed, it has the value 96 for both of the rings in (5.1).

\section{APPENDIX}

We give a list of all spaces of orderings, with accompanying information, for spaces with $|X| \leqslant 12$. These have been computer generated and are available up to $|X|=24$; the total number of distinct $(X, G)$ for each $|X|$ is computed in [8], where it is also pointed out that it suffices to list the even numbers. The construction is done in [7], where it is proved that each space $(X, G)$ for $|X|$ odd occurs by taking a space with one less point, adding a single point to the space and another square class to $G$; the associated $Y$ increases by one point, the covering number increases by 1 and the ring $W(X, G)$ is obtained as a product with a copy of $\mathbb{Z}$; the product here is in the category of Witt rings and an explicit construction is given in [7]. 


\begin{tabular}{|c|c|c|c|c|c|}
\hline$|X|$ & $\log _{2}|G|$ & $|Y|$ & $W(X, G)$ & covering no. & Comment \\
\hline 1 & 1 & 1 & $\mathbb{Z}$ & 1 & SAP, Fan \\
\hline 2 & 2 & 2 & $\mathbb{Z}^{2}$ & 2 & SAP, Fan \\
\hline 4 & 4 & 4 & $\mathbb{Z}^{4}$ & 4 & SAP \\
\hline$\overline{4}$ & 3 & 8 & $\mathbb{Z}[x, y]$ & 1 & Fan \\
\hline 6 & 6 & 6 & $\mathbb{Z}^{6}$ & 6 & SAP \\
\hline 6 & 5 & 10 & $\mathbb{Z}[x, y] \times \mathbb{Z}^{2}$ & 3 & \\
\hline 6 & 4 & 18 & $\mathbb{Z}^{3}[x]$ & 2 & \\
\hline 8 & 8 & 8 & $\mathbb{Z}^{8}$ & 8 & SAP \\
\hline 8 & 7 & 12 & $\mathbb{Z}[x, y] \times \mathbb{Z}^{4}$ & 5 & \\
\hline 8 & 6 & 16 & $(\mathbb{Z}[x, y])^{2}$ & 2 & \\
\hline 8 & 6 & 20 & $\mathbb{Z}^{3}[x] \times \mathbb{Z}^{2}$ & $\overline{4}$ & \\
\hline 8 & 5 & 32 & $\mathbb{Z}^{4}[x]$ & 2 & \\
\hline 8 & 4 & 128 & $\mathbb{Z}[x, y, z]$ & 1 & Fan \\
\hline 10 & 10 & 10 & $\mathbb{Z}^{10}$ & 10 & SAP \\
\hline 10 & 9 & 14 & $\mathbb{Z}[x, y] \times \mathbb{Z}^{6}$ & 7 & \\
\hline 10 & 8 & 18 & $(\mathbb{Z}[x, y])^{2} \times \mathbb{Z}^{2}$ & 4 & \\
\hline 10 & 8 & 22 & $\mathbb{Z}^{3}[x] \times \mathbb{Z}^{4}$ & 6 & \\
\hline 10 & 7 & 26 & $\mathbb{Z}^{3}[x] \times \mathbb{Z}[y, z]$ & 3 & \\
\hline 10 & 7 & 34 & $\mathbb{Z}^{4}[x] \times \mathbb{Z}^{2}$ & 4 & \\
\hline 10 & 6 & 50 & $\mathbb{Z}^{5}[x]$ & 3 & \\
\hline 10 & 6 & 130 & $\mathbb{Z}[x, y, z] \times \mathbb{Z}^{2}$ & 3 & \\
\hline 10 & 5 & 162 & $\overline{(\mathbb{Z} \times \mathbb{Z}[x, y])[z]}$ & 1 & \\
\hline 12 & 12 & 12 & $\mathbb{Z}^{12}$ & 12 & SAP \\
\hline 12 & 11 & 16 & $\mathbb{Z}[x, y] \times \mathbb{Z}^{8}$ & 9 & \\
\hline 12 & 10 & 20 & $(\mathbb{Z}[x, y])^{2} \times \mathbb{Z}^{4}$ & 6 & \\
\hline 12 & 10 & 24 & $\mathbb{Z}^{3}[x] \times \mathbb{Z}^{6}$ & 8 & \\
\hline 12 & 9 & 24 & $(\mathbb{Z}[x, y])^{3}$ & 3 & \\
\hline 12 & 9 & 28 & $\mathbb{Z}^{3} \times \mathbb{Z}[y, z] \times \mathbb{Z}^{2}$ & 5 & \\
\hline 12 & 9 & 36 & $\mathbb{Z}^{4}[x] \times \mathbb{Z}^{4}$ & 6 & \\
\hline 12 & 8 & 36 & $\left(\mathbb{Z}^{3}[x]\right)^{2}$ & 4 & \\
\hline 12 & 8 & 40 & $\mathbb{Z}^{4}[x] \times \mathbb{Z}[y, z]$ & 3 & \\
\hline 12 & 8 & 52 & $\mathbb{Z}^{5}[x] \times \mathbb{Z}^{2}$ & 5 & \\
\hline 12 & 7 & 72 & $\mathbb{Z}^{6}[x]$ & 3 & \\
\hline 12 & 8 & 132 & $\mathbb{Z}[x, y, z] \times \mathbb{Z}^{4}$ & 5 & \\
\hline 12 & 7 & 136 & $\mathbb{Z}[x, y, z] \times \mathbb{Z}[v, w]$ & 2 & \\
\hline 12 & 7 & 164 & $(\mathbb{Z}[x, y] \times \mathbb{Z})[z] \times \mathbb{Z}^{2}$ & 3 & \\
\hline 12 & 6 & 200 & $\left(\mathbb{Z}[x, y] \times \mathbb{Z}^{2}\right)[z]$ & 2 & \\
\hline 12 & 5 & 648 & $\mathbb{Z}^{3}[x, y]$ & 1 & \\
\hline
\end{tabular}




\section{REFERENCES}

[1] C. Andradas, L. Bröcker and J. Ruiz, Constructible sets in real geometry (Springer Verlag, Berlin, 1966).

[2] R. Baer, Linear algebra and projective geometry (Academic Press, New York, 1952).

[3] E. Becker and E. Köpping, 'Reduzierte quadratische Formen und semiornungen reeller Körpern', Abh. Math. Sem. Univ. Hamburg 46 (1977), 143-177.

[4] L. Bröcker, 'Zur Theorie der quadratischen Formen über formal reellen Körpern', Math. Ann. 210 (1974), 233-256.

[5] P.M. Cohn, Skew fields, Encyclopedia of Mathematics and its Applications 57 (Cambridge Univ. Presspubladdr Cambridge, 1995).

[6] T. Craven, 'The Boolean space of orderings of a field', Trans. Amer. Math. Soc. 209 (1975), 225-235.

[7] T. Craven, 'Characterizing reduced Witt rings of fields', J. Algebra 53 (1978), 68-77.

[8] T. Craven, 'An application of Pólya's theory of counting to an enumeration problem arising in quadratic form theory', J. Combin. Theory Ser. A 29 (1980), 174-181.

[9] T. Craven, 'Orderings and valuations on *-fields', Rocky Mountain J. Math. 19 (1989), 629-646.

[10] T. Craven, 'Witt groups of hermitian forms over *-fields', J. Algebra 147 (1992), 96-127.

[11] T. Craven, '*-valuations and hermitian forms on skew fields', in Valuation Theory and its Applications 1, Fields Inst. Commun. (American Mathematical Society, Providence, RI, 2002).

[12] T. Craven and T. Smith, 'Formally real fields from a Galois theoretic perspective', $J$. Pure Appl. Alg. 145 (2000), 19-36.

[13] T. Craven and T. Smith, 'Hermitian forms over ordered *-fields', J. Algebra 216 (1999), 86-104.

[14] T. Craven and T. Smith, 'Semiorderings and Witt rings', Bull. Austral. Math. Soc. 67 (2003), 329-341.

[15] I. Efrat and D. Haran, 'On Galois groups over pythagorean semi-real closed fields', Israel J. Math. 85 (1994), 57-78.

[16] T. Jacobi and A. Prestel, 'Distinguished representations of strictly positive polynomials', J. Reine Angew. Math. 532 (2001), 223-235.

[17] . J. Kleinstein and A. Rosenberg, 'Signatures and semisignatures of abstract Witt rings and Witt rings of semilocal rings', Canad. J. Math. 30 (1978), 872-895.

[18] J. Kleinstein and A. Rosenberg, 'Succinct and representational Witt rings', Pacific J. Math. 86 (1980), 99-137.

[19] M. Knebusch, 'Generalization of a theorem of Artin-Pfister to arbitrary semilocal rings, and related topics', J. Algebra 36 (1975), 46-67.

[20] M. Knebusch, A. Rosenberg and R. Ware, 'Structure of Witt rings and quotients of abelian group rings', Amer. J. Math. 94 (1972), 119-155.

[21] T. Y. Lam, Orderings, valuations and quadratic forms, Conference Board of the Mathematical Sciences 52 (American Mathemtical Society, Providence, RI, 1983).

[22] M. Marshall, Abstract Witt rings Queen's Papers in Pure and Appl. Math. 57 (Queen's University, Kingston, Ontario, 1980). 
[23] M. Marshall, Spaces of orderings and abstract real spectra, Lecture Notes in Math. 1636 (Springer-Verlag, Berlin, 1996).

[24] J. Merzel, 'Quadratic forms over fields with finitely many orderings', Contemp. Math. 8 (1982), 185-229.

[25] A. Prestel, Lectures on formally real fields, Lecture Notes in Math. 1093 (Springer-Verlag, Berlin, 1984).

[26] A. Prestel and C. Delzell, Positive polynomials: from Hilbert's 17th problem to real algebra, London Math. Soc. Note Ser. 36 (Springer-Verlag, Berlin, 2001).

Department of Mathematics

University of Hawaii

Honolulu, HI 96822

United States of America

e-mail: tom@math.hawaii.edu
Department of Mathematical Sciences

University of Cincinnati

Cincinnati, OH 45221-0025

United States of America

e-mail: tsmith@math.uc.edu 